${ }^{1}$ Servicio de Medicina. Hospital San Juan de Dios.

Universidad de Chile, Santiago, Chile.

2Departamento de

Medicina Interna y

Unidad Docente Asociada

Hospital Dr. Sótero del

Río, Pontificia Universidad Católica de Chile, Santiago, Chile.

Servicio de Nefrologia,

Hospital Dr. Sótero del Río Santiago, Chile.

${ }^{4}$ Departamento de

Anatomía Patológica,

Hospital San Juan de Dios, Santiago, Chile.

aResidente Medicina

Interna, Universidad de Chile.

Recibido el 25 de enero de 2011, aceptado el 17 de marzo de 2012

Correspondencia a: Dr. Juan Carlos Said E-mail: Esaid@uc.c.

\section{Glomerulopatía colapsante}

\author{
JUAN CARLOS SAID ${ }^{1, \mathrm{a}}$, LUZ MARÍA LETELIER², ALEJANDRO GONZÁLEZ², \\ CRISTÓBAL ESCOBILLANA ${ }^{3}$, RAÚL PISANO ${ }^{4}$
}

\section{Collapsing glomerulopathy}

\begin{abstract}
Collapsing glomerulopathy is a cause of nephrotic syndrome with massive proteinuria secondary to podocyte proliferation and glomerular collapse. It is characterized by an almost inevitable progression to end stage renal failure, poor response to treatment and high post-transplant recurrence. Its frequency has increased in recent years due to its common association with Human Immunodeficiency Virus (HIV) infection and the growing recognition of new etiologic agents such as drugs and parvovirus B19. Therefore, it is a disease of growing interest for clinicians. The aim of this review is to update the clinical presentation, diagnosis, pathogenesis and therapeutic alternatives of this disease.
\end{abstract}

(Rev Med Chile 2012; 140: 1342-1346).

Key words: Glomerulopathies; HIV; Parvovirus.
$\mathrm{E}$ 1 término "glomerulopatía colapsante" (GC), fue utilizado inicialmente por Weiss en 1986, al reportar 6 pacientes, que cursaron con síndrome nefrótico (SN), insuficiencia renal rápidamente progresiva y colapso glomerular, todo ello bajo el sugerente título: “¿Una nueva entidad clinicopatológica?”'. Por otra parte, en la misma década, comenzaron a reportarse casos de una nefropatía de similares características a la descrita por Weiss, pero asociada la infección por virus de inmunodeficiencia humana $(\mathrm{VIH})^{2}$. De ahí en adelante, se agregarían diferentes agentes como potenciales causas, todo lo cual llevaría a plantear esta nueva entidad como una probable vía final común a diferentes noxas.

La GC es una enfermedad proliferativa definida por el colapso segmentario o global de la membrana basal glomerular, asociada a hipertrofia e hiperplasia de los podocitos y a enfermedad tubulointersticial severa. Fue descrita en los años setenta como "glomeruloesclerosis focal y segmentaria maligna”, dada su rápida progresión a la insuficiencia renal terminal (IRT) ${ }^{3}$.

Aunque era una causa poco frecuente de IRT, su prevalencia ha ido en aumento en los últimos años, tanto en forma absoluta, como en proporción respecto al total de causas de IRT. De hecho, su frecuencia ha aumentado en los centros de referencia de biopsias renales, reportándose en un estudio retrospectivo realizado en México, entre 1985 y 1995, 15\% de GC en biopsias descritas como glomerulopatía focal y segmentaria ${ }^{4}$, similar al 10\% reportado en Estados Unidos de Norteamérica $^{5}$, lo cual probablemente se relaciona con un mayor índice de sospecha, mayor número de biopsias y lógicamente, la expansión de la pandemia del SIDA ${ }^{6}$.

En Chile, se han reportado casos, pero no existen datos de su prevalencia ${ }^{7}$. En la Pontificia Universidad Católica de Chile, centro de referencia nacional en biopsias renales, la GC en su forma pura (VIH y no VIH) sigue siendo infrecuente en la casuística de biopsias, con 0 a 1 caso por año aproximadamente de un promedio de 400 biopsias renales/año, lo cual podría deberse al bajo índice de sospecha por parte de los clínicos y a una subutilización de la biopsia renal en los casos de síndrome nefrótico (Comunicación no publicada. Servicio de Anatomía Patológica, Pontificia Universidad Católica de Chile).

En los Estados Unidos de Norteamérica se estima en $3,5 \%$ a $6,9 \%$ de los pacientes VIH positivos, con una fuerte predilección racial ${ }^{2,8}$. Una serie de 102 pacientes con CG no asociada a VIH, mostró que $50 \%$ de los pacientes eran de origen africano, $33 \%$ blancos, $10 \%$ hispánicos y $7 \%$ otras 
razas, lo cual podría traducir cierta predisposición genética ${ }^{9}$. En los Estados Unidos de Norteamérica, en personas de raza negra entre 20 y 64 años, es la tercera causa de IRT luego de diabetes e hipertensión ${ }^{10}$.

El objetivo de esta revisión es describir y actualizar las formas más frecuentes de presentación de la GC, la etiopatogenia y las herramientas diagnósticas y terapéuticas desde una perspectiva clínica.

\section{Diagnóstico y etiología}

La GC debe considerarse dentro del diagnóstico diferencial de todo paciente con SN. Clínicamente se caracteriza por proteinuria en rango nefrótico (mayor a 3,5 g en 24 h), asociado a lipiduria y edema, sin hematuria ni hipertensión. En general cursa con proteinuria masiva (mayor a $10 \mathrm{~g}$, en $24 \mathrm{~h}$ ) y rápida evolución a IRT ${ }^{11}$.

El estudio de estos pacientes debe incluir exámenes básicos de función renal (nitrógeno ureico y creatinina en sangre), perfil lipídico, hemograma, orina completa y proteinuria $24 \mathrm{~h}$, asociado según sospecha clínica, al estudio etiológico habitual de un $\mathrm{SN}$, buscando descartar diabetes, mieloma múltiple y patologías autoinmunes, entre otras. En el examen de orina, la presencia de lipiduria y cilindros granulares es común a otras causas de $\mathrm{SN}$, pero destaca la presencia de proteinuria masiva, rasgo distintivo en relación a la glomeruloesclerosis focal y segmentaria (GFS) y otras causas de SN.

Aunque la GC es una enfermedad de causa desconocida, se asocia a diversas patologías o fármacos, que deben considerarse dentro del estudio diagnóstico. Se describe primordialmente asociada a $\mathrm{VIH}^{12}$, especialmente en pacientes con mal control y bajo recuento de CD4, sin embargo, también ha sido reportada como parte de una infección aguda o en pacientes con carga viral indetectable ${ }^{10}$. Se ha asociado también con otras enfermedades infecciosas como parvovirus B19 $9^{12}$ $\mathrm{y}$ menos frecuentemente con citomegalovirus, HTLV- $1^{11}$, virus hepatitis $C^{13}$ y tuberculosis ${ }^{14}$. Además se la ha asociado a algunas enfermedades autoinmunes como lupus y enfermedad de Still ${ }^{15}$.

En forma más esporádica, se reporta asociación con algunos medicamentos como interferon $\mathrm{Alfa}^{16}$ y bifosfonatos pero en el caso de estos últimos, sólo cuando son usados en dosis supraterapéuticas ${ }^{17}$.En frecuencia decreciente se ha descrito en pacientes post trasplante renal, asociado a neoplasias y de origen genético, siendo en todos los grupos más común entre población afroamericana, excepto en los relacionados a fármacos y en post trasplante donde se reportan más casos en población blanca ${ }^{18}$.

Dado lo anterior, debe realizarse una anamnesis completa, que incluya dirigidamente búsqueda de factores de riesgo para enfermedades de trasmisión sexual y síntomas asociados a patologías autoinmunes. Se debe también indagar exhaustivamente en el uso de fármacos, con o sin prescripción médica y antecedentes familiares y raciales.

Finalmente, como en todo SN, sin causa evidente, la biopsia renal resulta imprescindible para certificar el diagnóstico y establecer el pronóstico.

\section{Patología}

Los hallazgos morfológicos más relevantes de esta entidad se observan tanto a nivel de los glomérulos, como a nivel tubular. En tinción de plata se observa un colapso capilar, con hiperplasia, hipertrofia y desarrollo de pseudocrescentes que ocupan el espacio de Bowman ${ }^{9}$. Con microscopía electrónica, a nivel glomerular se evidencia la proliferación podocitaria y la pérdida de los procesos secundarios. Lo anterior, debido a desregulación de los mecanismos de diferenciación, evidenciados por la pérdida de marcadores inmunohistoquimícos clásicos del podocito, como el WT-1, y la adquisición de marcadores previamente inexistentes, como el CD68. Dicha proliferación, que puede ser global o segmentaria, lleva eventualmente al colapso del asa capilar, al alterarse la capacidad por parte de los procesos podocitarios de ofrecer un soporte adecuado a la membrana basal capilar subyacente. Esta se retrae y repliega sobre sí misma, produciendo un colapso parcial o total del ovillo, hacia el hilio glomerular con ensanchamiento del espacio de Bowman. Al colapsar el glomérulo, manifestado por fibrosis cicatricial, se observan cambios degenerativos y regenerativos micro quísticos tubulares ${ }^{6}$.

Del punto de vista morfológico, en un principio se clasificó como una variante de la GFS, concepto introducido por Detwiler et al, en $1994^{19}$. Sin embargo, la presencia de algunas características contrapuestas entre ambas patologías ha puesto en duda esta clasificación. Así, mientras la proliferación podocitaria caracteriza a la GC, la 
podocitopenia se relaciona con la GFS. En forma paralela, mientras en la GFS se han descrito mejorías con el implante de células madre o "stem cells" en modelos experimentales, la GC revierte en modelos experimentales con inhibidores de la proliferación.

Todo lo anterior ha llevado a plantear que podría tratarse de una entidad nosológica distinta y no sólo una variante de GFS.

\section{Patogenia}

Aunque la patogenia de la GC es aún desconocida, ya que no ha sido posible encontrar un mediador común que sea el factor desencadenante de todas las $\mathrm{GCs}^{20}$, la evidencia sugiere que la injuria primaria al podocito llevaría a una alteración del ciclo celular con una reversión hacia un patrón más inmaduro y la subsecuente proliferación podocitaria.

La asociación con patologías y agentes tan disímiles tendría en común la alteración de la homeostasis del sistema inmune, más específicamente, los linfocitos helper Tipo I, que ya se han asociado a otras patologías del parénquima renal ${ }^{9}$.

El posible componente genético de esta patología, se infiere de su alta frecuencia en población de raza negra y de la descripción de series con manifiesta agregación familiar ${ }^{21}$.

Por otra parte, aun cuando se asocia a VIH y/o raza negra, destaca un reporte de pacientes caucásicos VIH negativos en tratamiento por cáncer de mama o mieloma (sin nefropatía asociada a mieloma), con pamidronato en dosis mayores a las aprobadas por la $\mathrm{FDA}^{17}$, que desarrollaron GC. En estos casos se ha planteado como fisiopatología un malfuncionamiento mitocondrial, avalado en la detección de una proteína mitocondrial prenyltransferasa-like en modelo de ratones $\mathrm{KD} / \mathrm{KD}$ que desarrollan siempre la enfermedad. Esta hipótesis podría explicar la toxicidad por los bifosfonatos, ya que también se asociarían con una injuria a nivel mitocondrial ${ }^{22}$, sin embargo, permanece aún sin explicación, cómo se produce la reacción podocitaria frente a este mal funcionamiento mitocondrial ${ }^{17}$.

Por otra parte, la asociación con infecciones ha hecho plantear un efecto tóxico directo, por ejemplo, asociado a la infección por VIH o un efecto citopático indirecto mediado por citoquinas inflamatorias.

\section{Tratamiento y pronóstico}

La terapia puede dividirse en tres: el manejo general de un paciente con $\mathrm{SN}$, las terapias específicas en caso de identificarse un agente etiológico y las terapias dirigidas a la GC propiamente tal sin agente etiológico identificado.

\section{Medidas generales}

Se recomienda el uso inhibidores de la enzima convertidora de angiotensina (IECA) como elemento esencial en el control de la proteinuria, basado en estudios de cohorte prospectivos con GC asociada a VIH, que muestran una disminución de la progresión de la enfermedad asociado a uso de IECA ${ }^{23,24}$. En forma paralela, debe considerarse un control óptimo de la presión arterial, uso de espironolactona y manejo de la dislipidemia como formas probadas de enlentecer el deterioro de la función renal en paciente con $\mathrm{SN}$, aun cuando la evidencia de su efectividad para la GC en particular es escasa.

\section{Con agente etiológico identificado}

Debe iniciarse la terapia específica para la patología identificada. En la GC asociada a VIH, la evidencia, derivada de estudios observacionales, sugiere que el tratamiento es fundamentalmente el de la enfermedad de base mediante terapia antirretroviral (TARV). Así, los casos de IRT asociada a VIH aumentaron en Estados Unidos de Norteamérica progresivamente previo a la incorporación de TARV, para finalmente estabilizarse y declinar con la incorporación de TARV ${ }^{25}$. Más evidencia indirecta, se ha obtenido de estudios como SMART, donde el uso intermitente de TARV según nivel de CD4 comparado con uso continuo, se asoció a mayor incidencia de IRT ${ }^{26}$.

Dado lo anterior, y tomando en cuenta la imposibilidad ética de hacer estudios aleatorios que incorporen pacientes a TARV o placebo, se recomienda el uso de TARV independiente de la carga viral y el nivel de CD4 en todos los pacientes con GC asociada a $\mathrm{VIH}^{10}$. El uso de corticoides se encuentra avalado por diversos estudios observacionales, sin embargo, continúa siendo un tratamiento de segunda línea reservado para el subgrupo de pacientes que no responden a TARV. Se recomienda en dosis de $1 \mathrm{mg}$ por $\mathrm{kg}$ por dos meses titulando luego a la baja por dos meses ${ }^{27}$.

Se han descrito casos de remisión completa de 
la GC luego de tratar dirigidamente la patología asociada (Ej.: tuberculosis, mieloma, parvovirus B19) ${ }^{15,28}$. En el caso de GC relacionada con parvovirus $\mathrm{B} 19$, el tratamiento -basado en reporte de casos- parece ser el uso de inmunoglobulinas endovenosas. Todo lo anterior enfatiza la importancia de buscar exhaustivamente etiologías de base tratables, especialmente infecciosas, para no dar un tratamiento inmunosupresor, a quien probablemente se beneficie de un tratamiento potenciador del sistema inmune como las inmunoglobulinas ${ }^{29}$.

\section{Sin agente etiológico identificado}

En pacientes en los cuales no se identifica un agente causal, series retrospectivas que evalúan diferentes combinaciones de ciclofosfamida, ciclosporina y corticoides $^{30}$, han mostrado que la enfermedad es relativamente resistente al tratamiento inmunosupresor, con una baja tasa de remisión de la enfermedad renal y progresión frecuente a la IRT. Sin embargo, existen incluso en Chile, casos de pacientes que habrían tenido remisión con lisinopril y esteroides sintéticos con un seguimiento a 3 años ${ }^{7}$. Inmunomoduladores como el rituximab están en experimentación en otras podocitopatías y podrían llegar a ser de utilidad en las $\mathrm{GC}^{31}$. Dado lo anterior, en pacientes con GC sin ninguna asociación a enfermedad infecciosa luego de realizado un estudio exhaustivo, es razonable realizar una prueba terapéutica con corticoides.

Finalmente, se cuenta con el transplante renal como última opción, lo cual sin embargo, se complica por la posibilidad de recurrencia en el órgano transplantado ${ }^{18}$.

\section{Conclusiones}

La GC es una causa de SN de muy mal pronóstico. La expansión del VIH y el reconocimiento de nuevos agentes etiológicos la han convertido en una patología de interés creciente para el clínico, quien deberá sospecharla frente a SN con proteinuria masiva y confirmarla con biopsia renal.

En el futuro, queda por dilucidar la etiopatogenia de la enfermedad, más allá de sus múltiples asociaciones, con una vía común que vincule a todos los causantes de GC. Lo anterior, es de esperar, llevará al desarrollo de nuevas terapias basadas en la evidencia y al reconocimiento del carácter distintivo de esta enfermedad.

\section{Referencias}

1. Weiss MA, Daquioag E, Margolin EG, Pollak VE. Nephrotic syndrome, progressive irreversible renal failure, and glomerular "collapse": a new clinicopathologic entity? Am J Kidney Dis 1986; 7: 20-8.

2. Rao TK, Filippone EJ, Nicastri AD, Landesman SH, Frank E, Chen CK, et al. Associated focal and segmental glomerulosclerosis in the acquired immunodeficiency syndrome. N Engl J Med 1984; 310: 619-73.

3. Brown CB, Cameron JS, Turner DR, Chantler C, Ogg CS, Williams DG, et al. Focal segmental glomerulosclerosis with rapid decline in renal function ("malignant FSGS"). Clin Nephrol 1978; 10: 51-61.

4. Ávila Casado MC. Glomerulopatía colapsante: Una nueva entidad asociada a síndrome nefrótico e insuficiencia renal Terminal. Rev Invest Clín 1999; 51 (6): 367-73.

5. Crary GS, Gulbahce HE, Crosson JT, Collapsing Glomerulopathy: Asisitida systematic five year retrospective hystologic and inmunohistochemical study. J Am Soc Nephrol 1996; (7): 1772.

6. Albaqumi M, Barisoni L. Current Views on Collapsing Glomerulopathy. J Am Soc Nephrol 2008; 19: 127681.

7. Vega , Guarda F, Goecke H, Méndez GA Complete remission of non-HIV collapsing glomerulopathy with deflazacort and lisinopril in an adult patient. Clin Exp Nephrol 2010; 14: 385-8.

8. Ahuja TS, Borucki M, Funtanilla M, Shahinian V, Hollander M, Rajaraman S. Is the prevalence of HIVassociated nephropathy decreasing? Am J Nephrol 1999; 19: 655-9.

9. Albaqumi M, Soos TJ, Barisoni L, Nelso PJ. Collapsing Glomerulopathy. J Am Soc Nephrol 2006; 17: 2854-63.

10. Choi AI; O’Hare AM; Rodríguez R. Update on HIVassociated Nephropathy 2007; (5) en www.nephrologyrounds.com.

11. Laurinavicius A, Hurwitz S, Rennke HG. Collapsing glomerulopathy in HIV and non-HIV patients: a clinicopathological and follow up study. Kidney Int 1999; 56: 2203-13.

12. Schwimmerv JA, Markowitz GS, Valeri A, Appel GB. Collapsing Glomerulopathy. Semin Nephrol 2003; 23: 209-18.

13. Liapis H, Young D. Quiz Page. Am J Kidney Dis 2007; 49: 49-51.

14. Rodrigues CE, Sette LH, Torritani J, Malheiros DM, Titan SM, Barros RT, et al. Tuberculosis-associated collapsing glomerulopathy: remission after treatment. Ren Fail, 2010; 32: 143-6. 
15. Kumar S, Sheaff M, Yaqoob M, Collapsing Glomerulopathy in Adult Still's Disease. Am J Kidney Dis 2004; 43 (5): 4-10.

16. Kanungo S, Tamirisa S, Gopalakrishnan R, SalinasMadrigal 1, Bastani B. Collapsing glomerulopathy as a complication of interferon therapy for hepatitis C infection. Int Urol Nephro 2010; 42 (1): 219-22.

17. Markowitz GS, Appel GB, Fine PL, Fenves AZ, Loon NR, Jagannath $S$, et al. Collapsing Focal Segmental Glomerulosclerosis Following Treatment with High-Dose Pamidronate. J Am Soc Nephrol 2001; 12: 1164-72.

18. Toth CM, Pascual M, Williams WW. Recurrent collapsing glomerulopathy. Transplantation 1998; 65: 1009-10.

19. Detwiler RK, Falk RJ, Hogan SL, Jennette JC. Collapsing glomerulopathy: a clinically and pathologically distinct variant of focal segmental glomerulosclerosis. Kidney Int 1994; 45: 1416-24.

20. Herman E, Klotman P. HIV -Associated Nephropathy: Epidemiology, Pathogenesis and Treatment. Semin Nephrol 2003; 23: 200-20.

21. Ávila-Casado MC, Vargas-Alarcón G, Soto ME, Hernández G, Reyes PA, Herrera-Acosta J. Familial collapsing glomerulopathy: Clinical, pathological and immunogenetic features. Kidney Int 2003; 63: 233-9.

22. Szczech LA, Gupta SK, Habash R, Guasch A, Kalayjian $\mathrm{R}$, Appel R, et al. The Clinical epidemiology and course of the spectrum of renal disesases associated with HIV infection. Kidney Int 2004; 66: 1145-52.

23. Wei A, Burns GC, Williams BA, Mohammed NB, Visintainer P, Sivak SL. Longterm renal survival in HIVassociated nephropathy with angiotensin-converting enzyme inhibition. Kidney Int 2003; 64 (4): 1462-71.
24. Yah aya I, Uth man AO, Uth man MM. Interventions for HIV-associated nephropathy. Codhrane Database Syst Rev 200; 7 (4): CD007183.

25. National Institutes of Health, National Institute of Diabetes and Digestive and Kidney Diseases. U.S. Renal Data System (USRDS) Annual Data Report. Bethesda, 2001. http://www.usrds.org

26. Lundgren JD, Babiker A, El-Sadr W, Emery S, Grund B; Neaton JD, et al. Inferior clinical outcome of the CD4 $(+)$ cell count guided antiretroviral treatment interruption strategy in the SMART study: Role of CD4 (+) cell counts and HIV RNA levels during follow-up. J Infect Dis 2008; 197 (8): 1145-55.

27. Eustace JA, Nuermberger E, Choi M, Scheel PJ Jr, Moore $\mathrm{R}$, Briggs WA. Cohort study of the treatment of severe HIV-associated nephropathy with corticosteroids. Kidney Int 2000; 58 (3): 1253-60.

28. Shah S, Cavenagh J, Sheaf M, Thuraisingham RC. Remission of Collapsing Focal Segmental Glomerulosclerosis Following Chemotherapy for Myeloma. Am J Kidney Dis 2004; 43 (2): 10-2.

29. Moudgil A, Shidban H, Nast CC, Bagga A, Aswad S, Graham SL, at al. Parvovirus B19 infection-related complications in renal transplant recipients: treatment with intravenous immunoglobulin. Transplantation 199; 64: 1847-50.

30. Stokes MB, Valeri AM, Markowitz GS, D’Agati VD. Cellular focal segmental glomerulosclerosis: Clinical and pathologic features. Kidney Int 2006; 70: 1783-92.

31. Marasà M, Kopp JB. Monoclonal antibodies for podocytopathies: rationale and clinical responses. Nat Rev Nephrol 2009; 5: 337-48. 